\title{
The carotenoid biosynthesis pathway in the asexual intraerythrocytic stages of Plasmodium falciparum
}

\author{
RICHARD NORTEY-MENSAH ${ }^{1}$, MARIAN A. TEYE ${ }^{1}$, MICHAEL F. OFORI ${ }^{2, \vartheta}$ \\ ${ }^{1}$ Department of Biochemistry, Cell and Molecular Biology, University of Ghana. Legon, Accra, Ghana \\ ${ }^{2}$ Noguchi Memorial Institute for Medical Research (NMIMR), University of Ghana. Legon, Accra, Ghana. "email: mofori@ noguchi.ug.edu.gh, \\ moforiori@noguchi.mimcom.org
}

Manuscript received: 30 November 2020. Revision accepted: 26 March 2021.

\begin{abstract}
Nortey-Mensah R, Teye MA, Ofori MF. 2021. The carotenoid biosynthesis pathway in the asexual intraerythrocytic stages of Plasmodium falciparum. Asian J Trop Biotechnol 18: 13-27. Plasmodium falciparum, like other Apicomplexans, possesses a dormant plastid called the apicoplast. Because it houses metabolic pathways peculiar to the parasite, such as isoprenoid production, this organelle promises a new target for the chemotherapeutic control of malaria. Although the phytoene synthase (PSY) gene has been shown to be critical for carotenogenesis; nothing is known about its evolutionary relationship with P. falciparum and other Apicomplexans. The goal of this work was to identify the evolutionary history and relatedness of the PSY gene in Apicomplexans and other animals, as well as to profile the carotenoids generated in the asexual intraerythrocytic stages of $P$. falciparum. Fluridone's IC50 and effect on parasite population were determined utilizing in vitro inhibition experiments on the asexual intraerythrocytic stages of $P$. falciparum. To examine the evolutionary history and relatedness of the PSY gene in Apicomplexans and other taxa, HPLC was used to profile carotenoids created at the asexual phases, and an unrooted phylogenetic tree was built using MEGA 6. Dose-dependent inhibition of parasite population was observed with fluridone treatment on all the asexual stages, with the ring stages being the most vuln erable. The carotenoid profiles revealed that carotenoids are generated cumulatively in $P$. falciparum throughout the asexual intraerythrocytic phases, with carotenoids such as lycopene, $\alpha$-, $\beta$-carotene among those synthesized. The discovery of relatively high quantities of abscisic acid (ABA) in the schizont stages, but not in the other stages was an interesting novel finding of this study. This is the first time ABA has been shown to be synthesized by $P$. falciparum, and additional research into the specific role of ABA in $P$. falciparum schizont phases would be groundbreaking. The phylogenetic study revealed that the $P$. falciparum PSY was most closely related to $P$. reichenowi, a chimpanzee strain of the malaria-causing parasites, supporting the hypothesis that malaria species in humans originated in chimps.
\end{abstract}

Keywords: Bioinformatics analysis, carotenoids, HPLC, in vitro, inhibition assays, Plasmodium falciparum

\section{INTRODUCTION}

Malaria is still one of the most serious parasite infections that affect humans, producing significant morbidity and mortality in tropical areas. According to the World Malaria Report, 198 million cases of malaria were reported worldwide, resulting in 580,000 fatalities (WHO 2014). Malaria is still endemic across five continents, despite ongoing and extensive attempts to suppress the illness. As a result, more than half of the world's population is at risk. Plasmodium parasites, of which there are five species, namely $P$. falciparum, $P$. vivax, $P$. ovale, $P$. malariae and $P$. knowlesi (Jiang et al. 2010; Sermwittayawong et al. 2012), cause human malaria, with the great majority of malaria-related deaths are caused by P. falciparum (Bousema and Drakeley 2011; Hay et al. 2009). The failure of vector control initiatives, the lack of a vaccine, and an increase in parasite resistance to routinely used therapeutic medicines have all contributed to the global spread of the disease (Sharma and Dutta 2011; Shiff 2002). The development of new chemotherapeutic techniques and medicines to attack Plasmodium necessitates a thorough understanding of the parasite's metabolic pathways. These new chemotherapeutic strategies and agents can be developed in one of three ways: (i) by developing new drug candidates based on previously validated parasite targets, (ii) by identifying new potential parasite targets for malaria chemotherapy, or (iii) by performing high-throughput drug library testing (Choi et al. 2008).

As in other apicomplexans (e. g. Toxoplasma spp.), Plasmodium parasites have retained a relic plastid organelle known as apicoplast which comes from plastidbearing red algae of secondary endosymbiosis millions of years ago (Foth and McFadden 2003). As a result, the apicoplast is prokaryotic in origin, containing pathways that do not exist in the human host. It possesses a genome that is like that of plants and algae, and according to proteome research, its metabolic processes include type II fatty acid and isoprenoid production (Sato 2011). A range of new plant-like enzymes has recently been found in malaria parasites, particularly the most virulent species, $P$. falciparum, with some of these being linked to the apicoplast (Kalanon and McFadden 2010). These enzymes are known to be part of many important biochemical pathways in plants and algae (Foth and McFadden 2003; Gornicki 2003; Seeber 2003). The carotenoid biosynthesis pathway is one of these metabolic pathways. It is an intriguing topic for research because it is required in algae, 
higher plants, bacteria, and fungi but not in mammals. The pathway's products are engaged in a variety of vital metabolic functions (Paniagua-Michel et al. 2012).

Plasmodium also retains biosynthesis of isoprenoids (resident in apicaloplast) resulting in the synthesis, by the pathway mevalonate-independency, of isopentenyl pyrophosphates (IPP) and dimethylallyl phosphates (DMAPP) (Jordão et al. 2011; Rodríguez-Concepción and Boronat 2013). These isoprene units serve as building blocks for the synthesis of isoprenoids, rather than being an end in themselves (for example, carotenoids). Fosmidomycin, an inhibitor of non-mevalonate isoprenoid precursor production in the apicoplast, has previously been shown to impede the proliferation of asexual blood-stage $P$. falciparum (van der Meer and Hirsch 2012; Wiesner et al. 2003). Supplementation with IPP, the pathway product, chemically reversed fosmidomycin inhibition in $P$. falciparum, according to Yeh and DeRisi (2011). In addition, abscisic acid (a product of the isoprenoid pathway) has been linked to the control of calciumdependent parasite egress in $T$. gondii, a similar apicomplexan, with suppression of its synthesis resulting in dose-dependent delays in parasite egress from the host cell (Nagamune et al. 2008). The isoprenoid biosynthetic pathway is required for the survival of apicomplexan parasites during their infective phases, according to these findings. Most of the downstream products of this pathway, as well as their roles in the asexual intraerythrocytic stages of $P$. falciparum parasites, are unclear currently.

In Plasmodium, various genes have been proposed to play a role in the carotenoid biosynthesis pathway, but only the phytoene synthase (PSY) gene (PfB0130w) has been definitively identified through functional experiments (Rodrguez-Villalón et al. 2009; Toledo-Ortiz et al. 2010). The phytoene synthase (PSY) gene encodes the enzyme phytoene synthase (PSY), which is a crucial protein because it catalyzes the first committed and rate-limiting step in the carotenoid biosynthesis pathway (Welsch et al. 2000) and acts as a key regulator of carbon inflow into the process. Rodrguez-Villalón et al. (2009) and Welsch et al. (2000) found that it catalyzes the condensation of two molecules of geranylgeranyl diphosphate (GGPP) to create phytoene, the basic C-40 carotenoid skeleton of all carotenoids. The PSY protein's structure (secondary and tertiary), function, and phylogenetic history in apicomplexans, plants, bacteria, and algae have all been studied (Tonhosolo et al. 2005; Agarwal et al. 2015). The evolutionary history and relatedness of PSY gene have been studied in other species. However, there are no studies on the gene's evolutionary history and relatedness in apicomplexans.

The specific goals of this study were to: (i) investigate the effect of fluridone on the asexual intraerythrocytic stages of $P$. falciparum using in vitro inhibition assays, (ii) to profile the carotenoids synthesized by the asexual intraerythrocytic stages of $P$. falciparum using HighPerformance Liquid Chromatography (HPLC), and (iii) to figure out the evolutionary history and relatedness of the phytoene synthase gene in $P$. falciparum and other organisms through bioinformatics analysis.

\section{MATERIALS AND METHODS}

All work relating to parasite culture was performed in a biological safety cabinet (BSC) using aseptic techniques.

\section{Media preparation and parasite culture \\ Preparation of media for culturing}

RPMI 1640 [with L-glutamine and HEPES (N-2hydroxyethyl piperazine-N'-2-ethane sulfonic acid)] (Gibco, UK) which was supplemented with $7.5 \%$ $\mathrm{NaHCO} 3$ (Sigma-Aldrich, USA) (working concentration, $32 \mathrm{mM}), 20 \%$ glucose (Sigma-Aldrich, USA) solution (working concentration, $20 \mathrm{mM}$ ), and gentamycin (Gibco, $\mathrm{UK}$ ) (working concentration, $10 \mathrm{~g} / \mathrm{ml}$ ) was used to generate $500 \mathrm{~mL}$ parasite washing media (PWM). PWM supplemented with 5mg/ml Albumax II (Invitrogen, USA) and $0.2 \mathrm{~g} / \mathrm{mL}$ hypoxanthine (Sigma- Aldrich, USA) was used to produce complete parasite medium (CPM). All of the culture mediums were kept between $4-8^{\circ} \mathrm{C}$ of temperature.

\section{Thawing of cryopreserved parasite}

Plasmodium falciparum 3D7 strain [which was cryopreserved and obtained from the Immunology Department of the Noguchi Memorial Institute for Medical Research (NMIMR), Ghana] was removed from liquid nitrogen storage and thawed in a $37^{\circ} \mathrm{C}$ water bath for 1 minute. After that, for every $100 \mathrm{~L}$ of parasite suspension, $12 \% \mathrm{NaCl}$ (Sigma-Aldrich, USA) was added. After a 5minute incubation period at room temperature, one-tenth of a parasite suspension in $1.6 \% \mathrm{NaCl}$ was introduced drop by drop to the tube while gently rotating it. At room temperature, the suspension was centrifuged for 5 minutes at 1500 revolutions per minute (rpm) (RT). The erythrocytes were resuspended in complete parasite medium (RPMI 1640 containing 5mg/mL Albumax II, $10 \mathrm{~g} / \mathrm{mL}$ gentamycin, $0.2 \mathrm{~g} / \mathrm{mL}$ hypoxanthine, $2 \mathrm{mM} \mathrm{L}-$ glutamine, $25 \mathrm{mM}$ HEPES, $23.8 \mathrm{mM} \mathrm{NaHCO} 3$ ) after the supernatant was removed. After that, the suspension was centrifuged at $1500 \mathrm{rpm}$ for 5 minutes at room temperature. The parasitized RBCs were added to 2001 of freshly generated sickling negative, $\mathrm{O}+$ human erythrocytes in a $25 \mathrm{ml}$ tissue culture flask (Corning, USA) containing $5 \mathrm{ml}$ CPM after the supernatant was removed.

\section{Parasite culture and maintenance}

Plasmodium falciparum 3D7 strain (NMIMR, Ghana) was cultured as described by Trager and Jensen (1976), with a few changes (Maier and Rug 2013). In either 25ml or $75 \mathrm{ml}$ tissue culture flasks, site cultures were maintained in $\mathrm{CPM}$ with human $\mathrm{O}+$, sickling negative erythrocytes at $4 \%$ hematocrit (Corning, USA). In $25 \mathrm{ml}$ and $75 \mathrm{ml}$ tissue culture flasks, five milliliters $(5 \mathrm{ml})$ or twenty-five milliliters $(25 \mathrm{ml})$ of CPM were employed to keep the cultures alive. The flasks were then gently flushed with the gas combination, firmly closed, and incubated at $37^{\circ} \mathrm{C}$. The RBCs utilized to sustain the cultures were obtained from a donor and placed in CPD vacutainers (BD biosciences, UK). Before processing, the blood was kept at $4-8^{\circ} \mathrm{C}$ for 48 hours. The blood was then placed into $15 \mathrm{ml}$ centrifuge 
tubes and washed three times with PWM by centrifuging at $1500 \mathrm{rpm}$ for five minutes and discarding the supernatant. After centrifuging for 5 minutes at room temperature in a swinging bucket rotor at $2,000 \mathrm{rpm}$, the hematocrit was calculated from the packed RBC volume. This was then refrigerated at $4-8^{\circ} \mathrm{C}$ for two weeks before being utilized for culture. Unless otherwise noted, spent CPMs were replaced with new ones, and the population and health of the cultures were monitored daily. By forming thin smears of the culture on a microscope slide, fixing them with methanol and staining them with $10 \%$ Giemsa (Fluka chemicals, UK) for 15 minutes, the parasite population was calculated. Parasitemia was determined by counting at least 1,000 erythrocytes with a 100 -fold oil-immersion objective to determine the percentage of infected erythrocytes. This technique was carried out daily.

\section{Synchronization of parasites}

$D$-Sorbitol synchronization for rings

With few changes, $P$. falciparum cultures were synchronized as described by Lambros and Vanderberg (1979). For synchronization, cultures with high ring stage parasite populations $(8 \%)$ were chosen. The selected cultures were placed into $15 \mathrm{ml}$ centrifuge tubes and spun at $2000 \mathrm{rpm}$ for five minutes to establish synchrony, with the supernatant discarded and the pellet resuspended in $5 \mathrm{ml}$ of aqueous $5 \%$ D-sorbitol solution pre-warmed to $37{ }^{\circ} \mathrm{C}$ in a water bath. The cell suspension was then cultured in a $37^{\circ} \mathrm{C}$ incubator for 10 minutes. The suspension was centrifuged at $2000 \mathrm{rpm}$ for 5 minutes after the incubation period, the D-sorbitol solution was removed, and the pellet was washed twice with PWM and once with CPM. All the washing stages took 5 minutes at $2000 \mathrm{rpm}$. A small smear from the cleaned cultures was put on a microscope slide, fixed with methanol, and stained with $10 \%$ Giemsa for 15 minutes. It was viewed with the $100 \mathrm{X}$ magnification objective lens assured successful synchronization. When the majority ( $90 \%$ or more) of the parasites spotted under the microscope were in the ring stages, synchronization was declared effective. The cleansed pellet was then transferred into a new $25 \mathrm{ml}$ tissue culture flask containing $5 \mathrm{ml} \mathrm{CPM}$ and uninfected $\mathrm{O}+$, sickling negative human RBCs at $4 \%$ hematocrit to start a new culture. The content of the flask was then gassed for 30 seconds with the specific gas mixture, sealed promptly and snugly, and put into an incubator at $37^{\circ} \mathrm{C}$ of temperature.

\section{Percoll $^{\circledR}$ synchronization for schizonts}

Nine parts of $100 \%$ Percoll ${ }^{\circledR}$ (Sigma-Aldrich, USA) were gently mixed with one part of $10 \mathrm{X}$ phosphatebuffered saline [(PBS), Gibco, USA] to make $90 \%$ Percoll® solution. By combining the $90 \%$ Percoll ${ }^{\circledR}$ solution with PWM, 65 percent $(65 \%)$ and 35 percent (35\%) Percoll@ solutions were produced. To make the 65 $\%$ Percoll@ solution, mix $6.5 \mathrm{ml}$ of $90 \%$ Percoll@ with $2.5 \mathrm{ml}$ of parasite wash medium (PWM), and mix $3.5 \mathrm{ml}$ of $90 \%$ Percoll@ with $5.5 \mathrm{ml}$ of PWM to generate $35 \%$ Percoll@ solution. After that, a $0.22 \mu \mathrm{m}$ filter (Millipore, France) was used to filter sterilize them. The purpose of these solutions was to establish a gradient. Meanwhile, the parasite cultures were placed into $15 \mathrm{ml}$ Fisherbrand centrifuge tubes, centrifuged at $2000 \mathrm{rpm}$ for 5 minutes at room temperature. The supernatant was then discarded and the pellet resuspended in PWM to a $10 \%$ hematocrit (total of about $2.5 \mathrm{ml})$. The Percoll@ gradient $(35 \% / 65 \%)$ was created by gently transferring $3 \mathrm{ml}$ of $65 \%$ Percoll® into a $15 \mathrm{ml}$ centrifuge tube and then gently transferring 3 $\mathrm{ml}$ of 35 percent Percoll ${ }^{\circledR}$ onto it along the tube's wall with a Pasteur pipette. The freshly generated Percoll ${ }^{\circledR}$ gradient was then carefully overlaid with the resuspended parasite culture $(2.5 \mathrm{ml})$. This was then centrifuged for 15 minutes at $2500 \mathrm{rpm}$ at room temperature without a break in a swing-out rotor. Parasites were retrieved from the 35-65\% interface after centrifugation, transferred to a new $15 \mathrm{ml}$ centrifuge tube, washed twice with PWM and once with CPM, and cultured at $6 \%$ hematocrit.

\section{Preparation and sensitivity assays of test compounds Preparation of test compounds}

Fluridone [test compound (Sigma-Aldrich, Germany)] dissolved in dimethyl sulfoxide [DMSO (Sigma-Aldrich, Germany)] and artemisinin [control drug (Sigma-Aldrich, USA)] were uniformly coated in flat-bottom 96-well microtitre plates (Thermo Scientific NuncTM, USA). Twofold serial dilutions of the test compounds were prepared in triplicates in the respective wells. For the sensitivity assays, the working concentrations for fluridone were $500 \mathrm{mM}$, $250 \mathrm{mM}, 125 \mathrm{mM}, 62.5 \mathrm{mM}, 31.25 \mathrm{mM}$, and $15.625 \mathrm{mM}$, while for artemisinin, they were $152 \mathrm{nM}, 76 \mathrm{nM}, 38 \mathrm{nM}$, $19 \mathrm{nM}, 9.5 \mathrm{nM}$, and $4.75 \mathrm{nM}$. Until they were ready to use, the drug-coated plates were kept at $-20^{\circ} \mathrm{C}$.

\section{Inhibition assays of test compounds against $\mathrm{P}$. falciparum asexual stages}

At $1 \%$ parasitemia and $2 \%$ hematocrit, in vitro inhibitory assays were performed for the asexual (rings, trophozoites, and schizonts) stages of P. falciparum 3D7 strain. The plates were put in an incubation chamber, gassed with a specific gas combination for 6 minutes, then incubated for 48 hours at $37^{\circ} \mathrm{C}$. Following the incubation period, 100L of a 1:10,000 SYGR Green I (Sigma-Aldrich, USA) solution in lysis buffer (20 nM Tris base, $5 \mathrm{mM}$ EDTA, $0.008 \%$ saponin, 0.08 percent Triton X-100, pH 7.5) was added to the cultures in the wells and carefully mixed. After that, the plates were incubated for an hour at room temperature in the dark. After incubation at excitation and emission wavelengths of $485 \mathrm{~nm}$ and $535 \mathrm{~nm}$, the fluorescence intensity of the cells in each well was measured using a fluorescent plate reader (Tecan endless 200pro, Japan).

\section{Extraction of carotenoids from $P$. falciparum asexual stages}

Plasmodium falciparum 3D7 parasites were cultivated in three $75 \mathrm{ml}$ tissue culture flasks for each asexual stage to generate a high ring stage population. To synchronize for the ring stage parasites, these cultures were treated with $5 \%$ D-sorbitol. At a parasitemia of $8-10 \%$, the synchronized cultures were allowed to proliferate to obtain rings, trophozoites, and schizonts. To obtain the cell pellet, the cultures at each stage were pooled and collected by 
centrifuging at $2000 \mathrm{rpm}$ for 5 minutes. The pellet was resuspended in $20 \mathrm{ml}$ of PBS, and the number of RBCs in the solution was determined using a hemocytometer. The parasitemia and RBC count were also used to estimate the number of infected RBCs. The number of parasites necessary for the extraction was set at 500 million. The cell pellets were obtained by centrifuging the pooled cultures at $3500 \mathrm{rpm}$ for 10 minutes at room temperature. The pellets were then resuspended in $10 \mathrm{ml}$ PBS with $0.1 \%$ saponin (0.007M Na2HPO4, 0.01M Na2HPO4, pH 7.4 and $0.15 \mathrm{M}$ $\mathrm{NaCl})$. The resuspended pellet was rinsed three times with PBS before being centrifuged for 10 minutes at $6000 \mathrm{rpm}$. When extraction could not be done right away, the pellets were kept at $-80^{\circ} \mathrm{C}$ until needed. The carotenoids were extracted by adding $1 \mathrm{~mL}$ of methanol to the parasite pellet, followed by $2 \mathrm{~mL}$ of hexane. After then, the suspension was vortexed for 2 minutes. After that, $1 \mathrm{ml}$ of water was added to the mixture, which was vortexed for another minute. This was then centrifuged for 20 minutes at 4,000 rpm. A $0.2 \mathrm{~m}$ nylon filter was used to capture and filter the supernatant phase. Following that, the filtrate was used straight for HPLC analysis.

\section{HPLC analysis of carotenoids extracted from $P$. falciparum asexual stages}

The HPLC analysis was carried out on a Shimadzu Prominence (Shimadzu Corp.) separation module outfitted with a Shimadzu Prominence (CTO-10ASV, Shimadzu Corp.) column oven, SIL-20AC HT auto sampler (Shimadzu Corp.), Shimadzu Prominence DGU-20A3 online degasser (Shimadzu Corp.), Shimadzu Prominence LC-20AB solvent delivery system (Shim (Shimadzu Corp.). The analytical scale AcclaimTM C30 reversedphase column employed $(250 \mathrm{~mm}$ x $4.6 \mathrm{~mm}$ internal diameter, $5 \mathrm{~m}$ particle size) was set at $25^{\circ} \mathrm{C}$. At a flow rate of $1 \mathrm{ml} / \mathrm{min}$, the mobile phase was a non-linear gradient of acetone/water with a starting composition of 50:50 (v/v). The overall run time was 45 minutes, and the filtrate injection volume was $20 \mu \mathrm{l}$. The eluents were detected using a UV-VIS detector with a wavelength of $475 \mathrm{~nm}$. To determine the identity of the resulted peaks, the retention durations of six carotenoid standards (lycopene, -carotene, -carotene, abscisic acid, lutein, and apo-carotenal) were calculated separately under the circumstances described above and compared to those obtained from the filtrate.

\section{Bioinformatics analysis of PSY PPS/O}

Nucleotide sequences of phytoene synthase $(P S Y)$ or octaprenyl pyrophosphate synthase (OPPS) from $P$. falciparum (PF3D7_0202700, PFB0130w) and other organisms were obtained from the gene databases of National Center for Biotechnology Information (NCBI, www.ncbi.nlm.nih.gov/gene/),

PlasmoDB (www.plasmodb.org/) and ToxoDB (www.toxodb.org/). Table S1 lists the primary accession numbers or gene IDs received for the PSY gene for the analysis. The $P$. falciparum OPPS/PSY gene sequence was utilized as a query for Basic Local Alignment Search Tool, nucleotide (BLASTn) (blast.ncbi.nlm.nih.gov) analysis to find homologs in other organisms. The best blast hits included
T. gondii and T. thermophilus octaprenyl pyrophosphate synthase. The nucleotide sequences were chosen from the BLAST result because the carotenoid production pathway has been extensively explored in these organisms.

\section{RESULTS AND DISCUSSION}

\section{In vitro inhibition assays}

Artemisinin and fluridone were used as control and test drugs in in vitro inhibitory experiments on the $P$. falciparum 3D7 strain, respectively. The inhibition assays were performed on the parasite's intraerythrocytic asexual stages (rings, trophozoites, and schizonts) to assess the effect of the compounds on the parasites as well as the halfmaximum inhibitory doses (IC50) against each stage. After 48 hours of incubation with the chemicals, the parasites were treated for an hour with a SYBR Green I in lysis buffer solution. The absorbance from the respective wells in the microtitre plates was measured with a fluorometer immediately after this incubation period. The fluorometer absorbance and chemical concentrations were put into Excel ${ }^{\circledR}$ spreadsheets and converted to $\%$ parasitemia and $\log$ concentrations, respectively. These were then entered into the GraphPad Prism ${ }^{\circledR}$ program (version 5) and a nonlinear regression was run to obtain dose-response curves and IC50 values for the drugs tested.

As percentage parasitemia is plotted on the y-axis against the log of concentration of the test chemicals on the $\mathrm{x}$-axis, a sigmoidal curve and a dose-dependent response are apparent in all the results from the inhibition assays performed. This was expected because high concentrations of the inhibitors, if effective against the parasite, will result in a higher level of inhibition of the targeted cellular process; resulting in a decrease in parasite population (lower parasitemia), if that biological process is essential for parasite survival, and vice versa. Figure 1 shows that fluridone therapy resulted in a gradual decline in the ring stage parasite population, with an $\mathrm{IC}_{50}$ of $17.00 \mathrm{mM}$. Treatment of the parasites with artemisinin (Figure 1) revealed a minimal response of the parasites to the first two lower concentrations of the compound, followed by a gradual decline in parasite population as the concentration of artemisinin increased. This resulted in an $\mathrm{IC}_{50}$ of 11.53 $\mathrm{nM}$ against the ring stages. When the concentrations at which fluridone and artemisinin attained their half-maximal inhibition were compared, artemisinin ( $\mathrm{IC}_{50}$ in nanomolar) demonstrated to be a far more effective inhibitor than fluridone, because its $\mathrm{IC}_{50}$ is substantially lower $\left(\mathrm{IC}_{50}\right.$ in millimolar). This was observed in all the inhibition trials for the other asexual stages, where half-maximal inhibition values of artemisinin were obtained at considerably lower doses than fluridone against the same stages. Fluridone inhibited growth at rates ranging from $43 \%$ to $62 \%$, while artemisinin inhibited growth at rates ranging from $55 \%$ to 75\% (Figure 1).

The inhibition experiments for both compounds on the parasite's trophozoite stages (Figure 2) were comparable, with essentially little response to the effects of both fluridone and artemisinin at lower dosages. Both 
compounds had a very flat line at the beginning of the graph (Figure 2). The slope of the graph for fluridone (Figure 2) increased sharply as the concentration of fluridone increased, resulting from a drop in percentage parasitemia. After treatment with the fourth-highest concentration of fluridone, the graph leveled off. After therapy with the sixth-highest concentration of artemisinin, the percentage of parasitemia begins to level off again.

As a result, additional increases in fluridone and artemisinin concentrations had no discernible effect on the parasite's trophozoite population. Fluridone treatment yielded a half maximum inhibitory concentration of $50.31 \mathrm{mM}$, while artemisinin therapy yielded a value of $9.67 \mathrm{nM}$. Fluridone inhibited growth by $40 \%$ to $60 \%$, while artemisinin inhibited growth by $50 \%$ to $70 \%$. Figure 3 depicts the results of the inhibition experiments against the parasite's schizont stages. At this stage of the parasite life cycle, both drugs tested had a dose-dependent effect. The graph for fluridone treatment reveals a dose-dependent

\section{I}

Fluridone Dose-Response curve for 3D7 rings

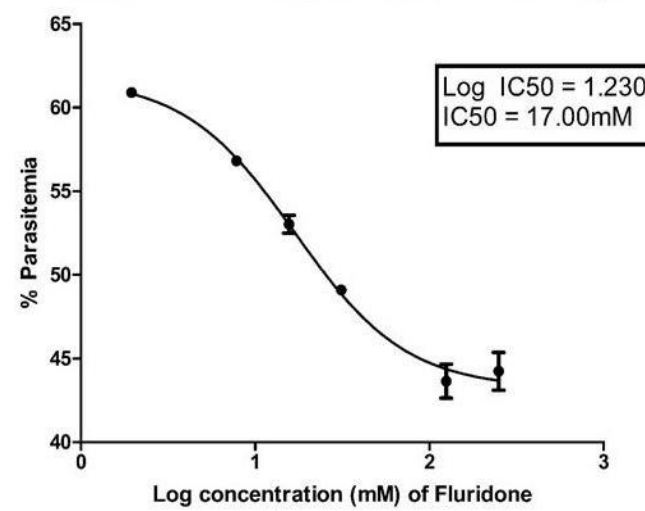

influence on the population of the parasite's schizont stages. From the beginning of the graph, there was a high slope. Fluridone's half-maximum inhibitory concentration was $25.10 \mathrm{mM}$, while fluridone's growth inhibition on the schizont stage ranged from $15 \%$ to $60 \%$.

In the instance of artemisinin (Figure 3), the compound's action can be seen even at the lowest concentration, when the parasite population begins to fall immediately, and this continues until the fourth-highest concentration, when the curve begins to level out. At 5.362 $\mathrm{nm}$, artemisinin reached its half maximal inhibitory concentration against the schizont stage. With regards to both drugs in this investigation, this was the lowest IC50 for the asexual phases. The percentage of growth inhibition ranged from 45 to $70 \%$. The ring stages were the most responsive to fluridone treatment, with an IC50 of $17.00 \mathrm{mM}$, almost three times and one-and-a-half times higher than the trophozoite $(50.31 \mathrm{mM})$ and schizont $(25.10 \mathrm{mM})$ stages, respectively.

\section{II}

\section{Artemisinin Dose-Response curve for 3D7 rings}

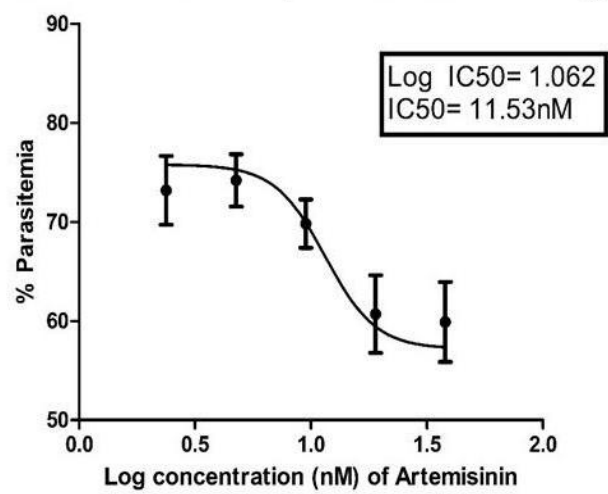

Figure 1. Dose-response curves for Plasmodium falciparum 3D7 ring stage parasites. Growth inhibitions by (I) fluridone and (II) artemisinin after incubating the parasites with different concentrations of these compounds for 48hrs. Error bars represent the standard error of mean (SEM) from triplicate readings of each concentration used.

\section{I}

Fluridone Dose-Response curve for 3D7 trophs

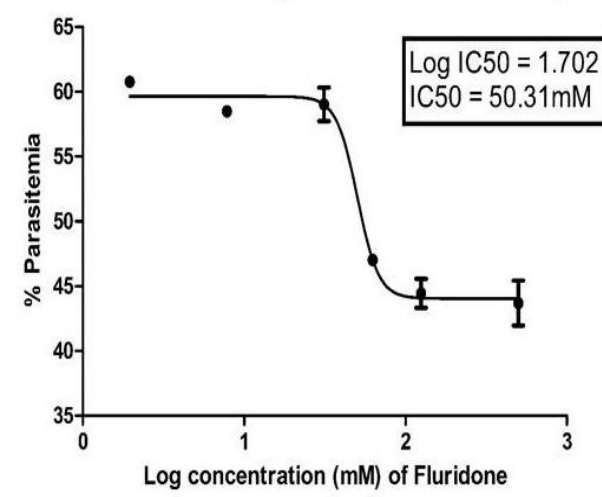

II

Artemisinin Dose-Response curve for 3D7 Trophs

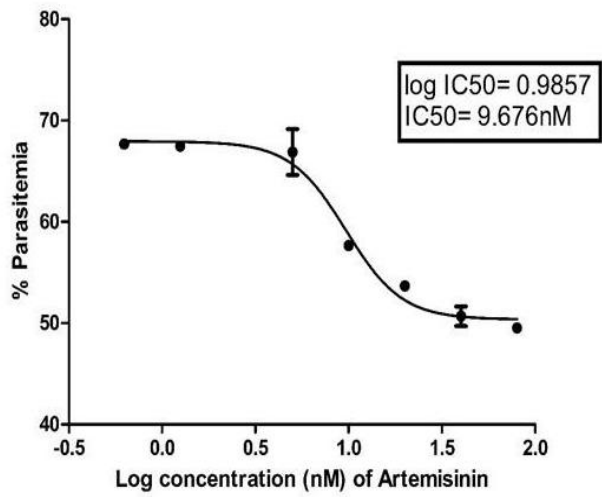

Figure 2. Dose-response curves for Plasmodium falciparum 3D7 trophozoite stage parasites. Growth inhibitions by (I) fluridone and (II) artemisinin after incubating the parasites with different concentrations of these compounds for 48hrs. Error bars represent the standard error of mean (SEM) from triplicate readings of each concentration used. 
I

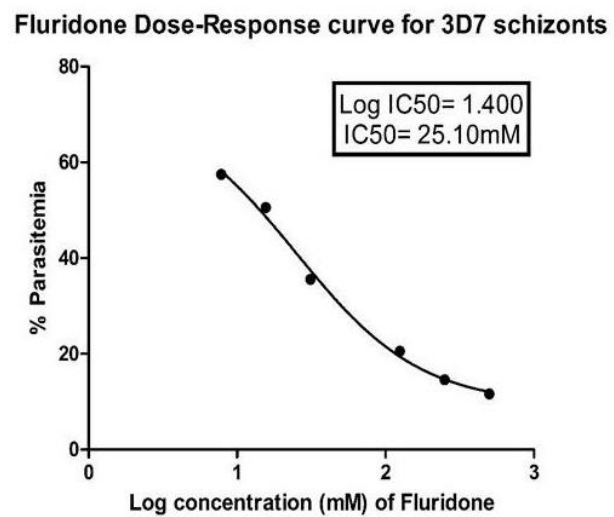

II

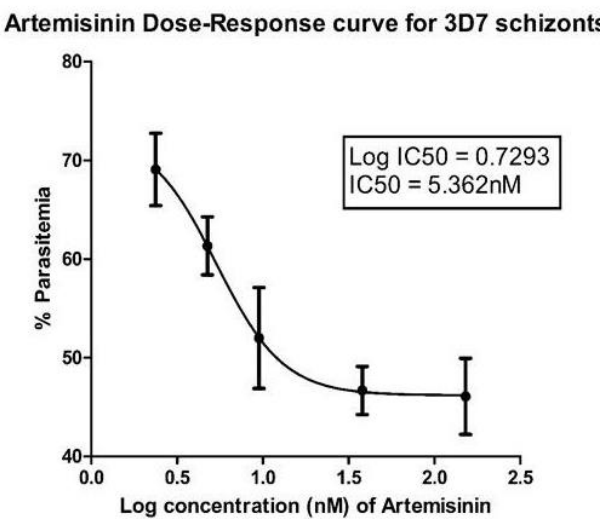

Figure 3. Dose-responses curve for Plasmodium falciparum 3D7 schizont stage parasites. Growth inhibitions by (I) fluridone and (II) artemisinin after incubating the parasites with different concentrations of these compounds. Error bars represent the standard error of mean (SEM) from triplicate readings of each concentration used.

\section{Carotenoid extraction and HPLC analyses}

Retention time is plotted on the $\mathrm{x}$-axis and milli Absorbance Unit (mAU) is plotted on the y-axis in the chromatograms (Figures 4, 5, and 6) shown below. There were few detected or observed peaks on the chromatogram of the extract from the ring stage parasites (Figure 4). The organic solvents (methanol and hexane) utilized for the extraction have peaks at the beginning of the chromatogram with retention durations between 2-4 minutes. At the outset of the run, they elute rather quickly. These peaks can also be seen in the trophozoite (Figure 5) and schizont (Figure 6) stage extraction chromatograms. The lone peak with a retention time of 22.754 minutes that can be seen farther down the chromatogram from the ring stage extract (Figure 4) is lutein. The peak was identified by comparing its retention time to the retention time of the standards employed in the investigation. The lutein concentration in the ring stage was determined to be $0.0032 \mathrm{mg} / \mathrm{ml}$. This was calculated using the HPLC machine's area under curve for each peak and the specific equation of line derived from the standard curves of the different carotenoid standards. Experiments that were duplicated yielded similar findings.

In comparison to the ring stages, the chromatogram for the trophozoite stage extraction (Figure 5) had a few more peaks. Aside from the initial peaks with retention periods of 2-4 minutes, three more peaks in the range of carotenoid retention durations were discovered. The peak's identification with a retention time of 17 . The duration of 763 minutes is unclear because it does not relate to any of the standards' retention times. With a retention duration of 19.573 minutes, the next peak was recognized as -carotene. The amount of -carotene generated by the trophozoite stage parasites was calculated to be $0.0031 \mathrm{mg} / \mathrm{ml}$. The last detectable peak (Figure 5) was recognized as carotene, with a retention period of 30.303 minutes and an estimated amount of $0.0019 \mathrm{mg} / \mathrm{ml}$. The carotenoids produced by trophozoite stage parasites were not the same as those produced by ring stage parasites. Duplicate experiments yielded similar results yet again.

There were multiple peaks on the chromatogram for the schizont stage extract (Figure 6). Some of these peaks had retention periods that matched the criteria, while others did not and so were not detected. The peaks detected at retention times of 3-5 minutes are, once again, those of the extraction solvents. 19.550 minutes ( $\alpha$-carotene), 23.567 minutes (apo-carotenal), 24.114 minutes (abscisic acid), 30.086 minutes (lycopene), and 30.348 minutes ( $\beta$ carotene) were the peaks having retention periods that corresponded to those of a standard utilized. The identity of the remaining peaks could not be validated because they did not have retention periods that corresponded to any of the criteria. In the schizont stage, the estimated levels of the detected carotenoids were $0.0824 \mathrm{mg} / \mathrm{ml}$ ( $\alpha$-carotene), $0.0032 \mathrm{mg} / \mathrm{ml}$ (apo-carotenal), $0.664 \mathrm{mg} / \mathrm{ml}$ (abscisic acid), $0.8321 \mathrm{mg} / \mathrm{ml}$ (lycopene), and $0.0022 \mathrm{mg} / \mathrm{ml}$ ( $\beta$-carotene). Experiments that were duplicated yielded similar findings. The largest peak in Figure 6 was recognized as abscisic acid. This indicates that high levels or quantities of this phytohormone are being generated, and it was only found during the parasite's schizont stage of life. As the parasites progressed from the young to the mature stages, the kind and quantity of the produced carotenoid clearly changed.

\section{Evolutionary history and phylogenetic analysis}

The phylogenetic link between the PSY gene of $P$. falciparum and homologs in other species is investigated (Figure 7). The PSY/OPPS gene sequence from $P$. falciparum was utilized as a query in a BLASTn search for homologs in other organisms. The PSY/OPPS sequences were chosen for the phylogenetic analysis using the BLAST results since the carotenoid production pathway has been extensively explored in these taxa. P. falciparum PSY was one of thirty PSY/OPPS gene sequences chosen from various taxonomic groups. Apicomplexans, fungi, plants, and bacteria were among the creatures chosen for the analysis. CLUSTAL W2 was used to align all the selected gene sequences, and MEGA6 was used to create an unrooted phylogenetic tree from the alignment using the Neighbor-Joining method. The numbers, along the branches, represent the evolutionary distance. These were calculated using the Maximum Composite Likelihood technique and was measured in number of base substitutions per site. 


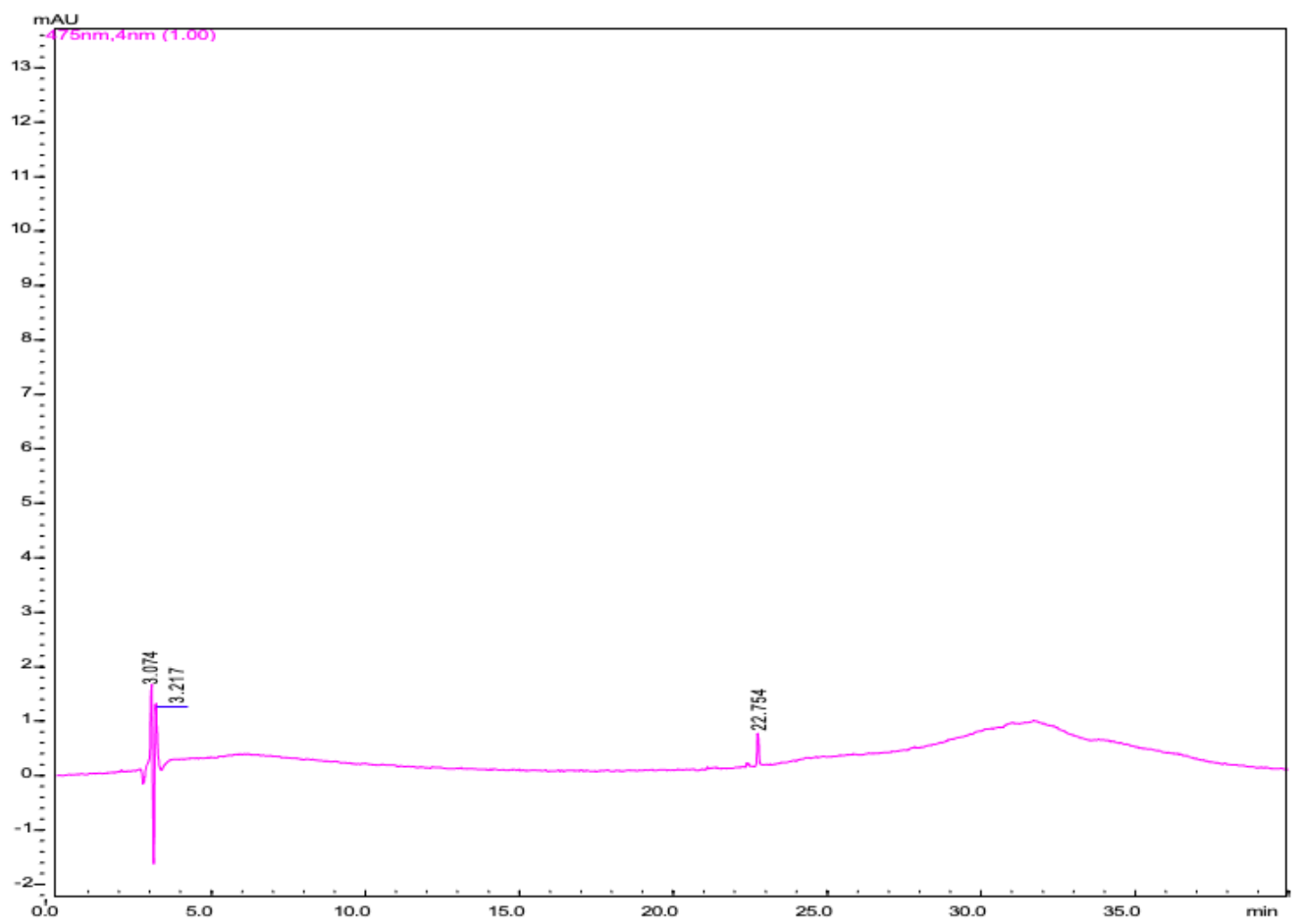

Figure 4. Chromatogram of carotenoids extracted from Plasmodium falciparum ring stage.

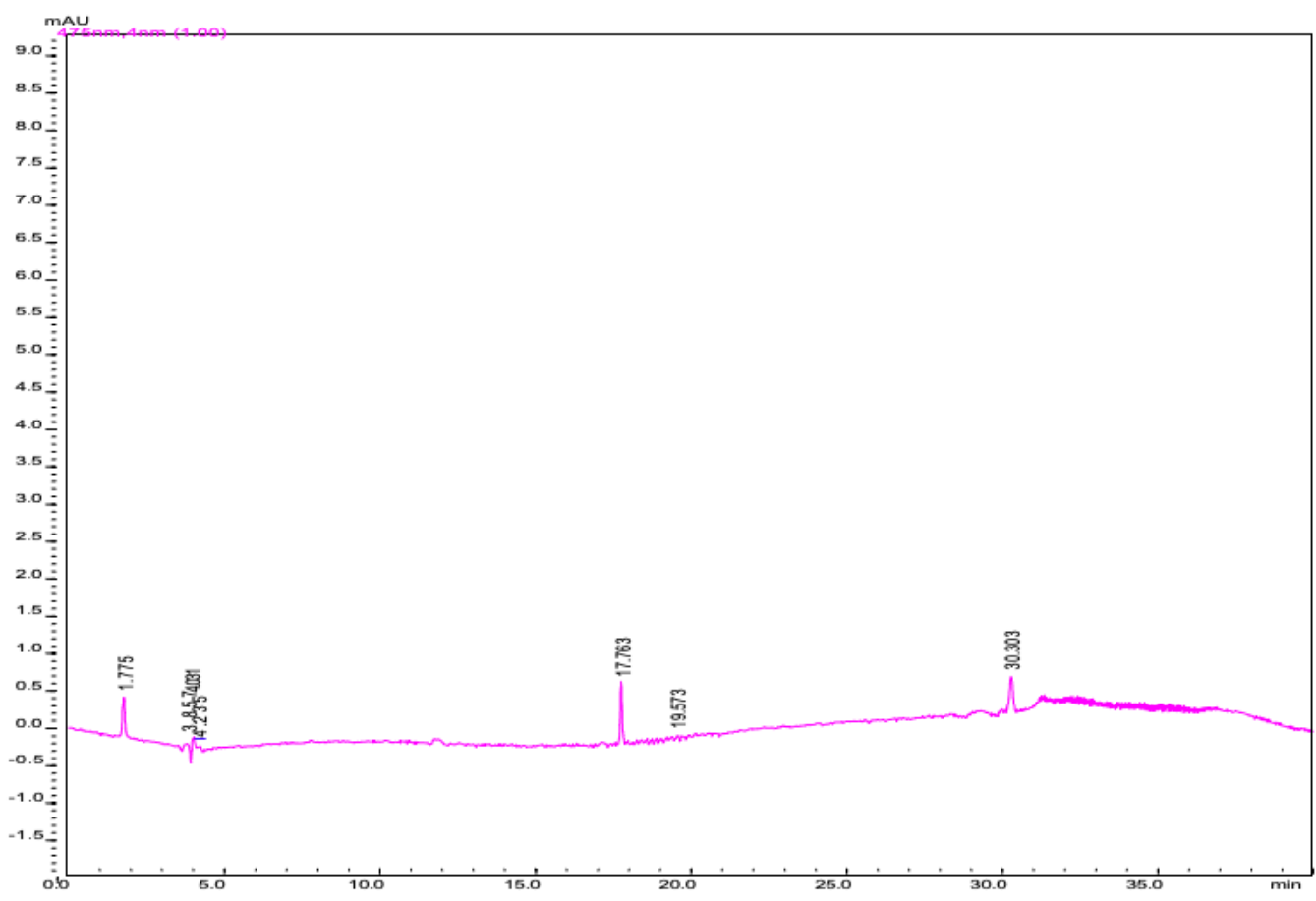

Figure 5. Chromatogram of carotenoid extracted from Plasmodium falciparum trophozoite stage. 


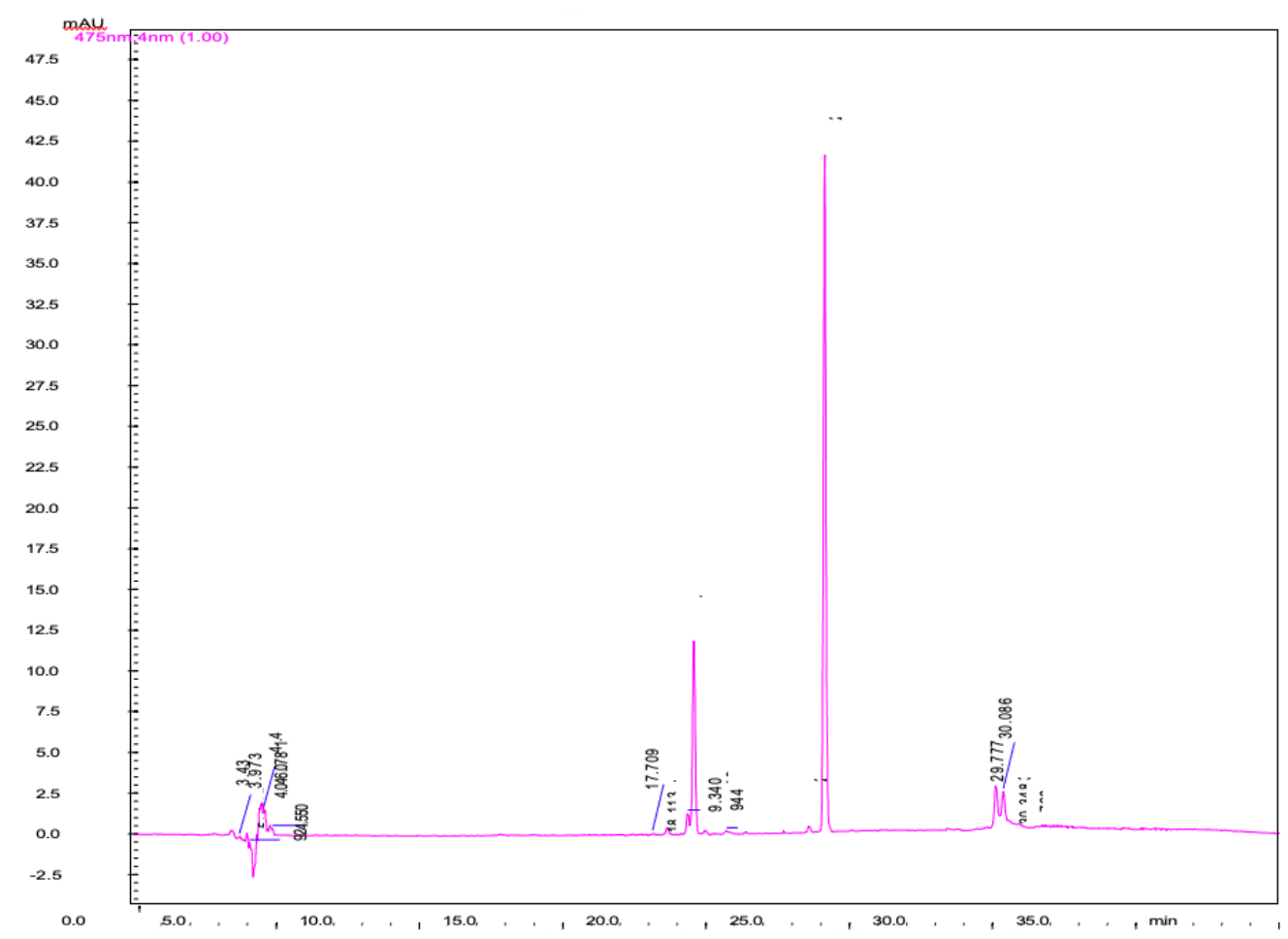

Figure 6. Chromatogram of carotenoid extracted from Plasmodium falciparum schizont stage. Carotenoids were extracted from synchronized cultures using a ternary solvent system (methanol/hexane/water) and the organic phase fraction was collected and run on a C- 30 column with a mobile phase of acetone/water for 40 minutes. Detection of the eluents was at $475 \mathrm{~nm}$.

On the unrooted tree, there are two major clades with multiple subclades branching out of each major clade (Figure 7). Plasmodium, Cryptosporidium, Theileria, Babesia, and Toxoplasma were found on one of the major clades, whereas Eimeria and the other apicomplexans were found on the other clades. As a result, the PSY gene in apicomplexans (yellow circle) split earlier in their evolution. The Plasmodium PSY gene forms a single subclade with 99 of bootstrap value, indicating a greater level of gene relatedness within this group and the likelihood that these species shared a common ancestor at some stage in their evolution. Surprisingly, $P$ falciparum established a subclade with $P$. reichenowi, a chimpanzee parasite, with 99 of bootstrap value, indicating that the $P$. falciparum PSY gene is most closely related to $P$. reichenowi among all the sequences utilized in this study.

The Neighbor-Joining approach was used to infer the evolutionary history. The ideal tree is presented, with a branch length sum of 15.24929587. Next to the branches are the percentage of duplicate trees in which the related taxa clustered together in the bootstrap test (100 repetitions). A total of 30 nucleotide sequences were examined. For each sequence pair, all unclear locations were deleted. The total number of places in the final dataset was 5432. MEGA 6 was used to conduct evolutionary analysis.

\section{Discussion}

In vitro inhibition assay

Over the last decade, efforts have been made in numerous areas, including vector control, vaccine development, and medicine research projects, to eradicate malaria from the world's impacted regions (malERA Consultative Group on Vaccines 2011; Raghavendra et al. 2011; Rieckmann 2006; Schwartz et al. 2012). The genetic plasticity of $P$. falciparum allows it to elude the host immune system (Jeffares et al. 2007; Wright and Rayner 2014) as well as develop resistance to some of the existing medications used to treat and control malaria (Djimdé et al. 2001; Dondorp et al. 2010; Schellenberg et al. 2006). More effort and resources must be directed toward vector management, vaccine development, and therapeutic research to win the battle against the illness. Currently, the hunt for a viable vaccine candidate to aid in the eradication of the illness is well underway (Casares et al. 2010; Crompton et al. 2010; Malik et al. 2012). Novel chemicals and their effects on new or established targets in $P$. falciparum metabolic pathways are also being studied (Crowther et al. 2011; Qidwai and Khan 2012). In apicomplexans, the apicoplast organelle is an attractive new target (Kalanon and McFadden 2010; Maréchal and Cesbron-Delauw 2001). In apicomplexans, the apicoplast organelle is an attractive new target (Kalanon and McFadden 2010; Maréchal and Cesbron-Delauw 2001). Metabolic processes such as fatty acid synthesis and carotenoid production are housed inside it (Seeber and Soldati-Favre 2010). 


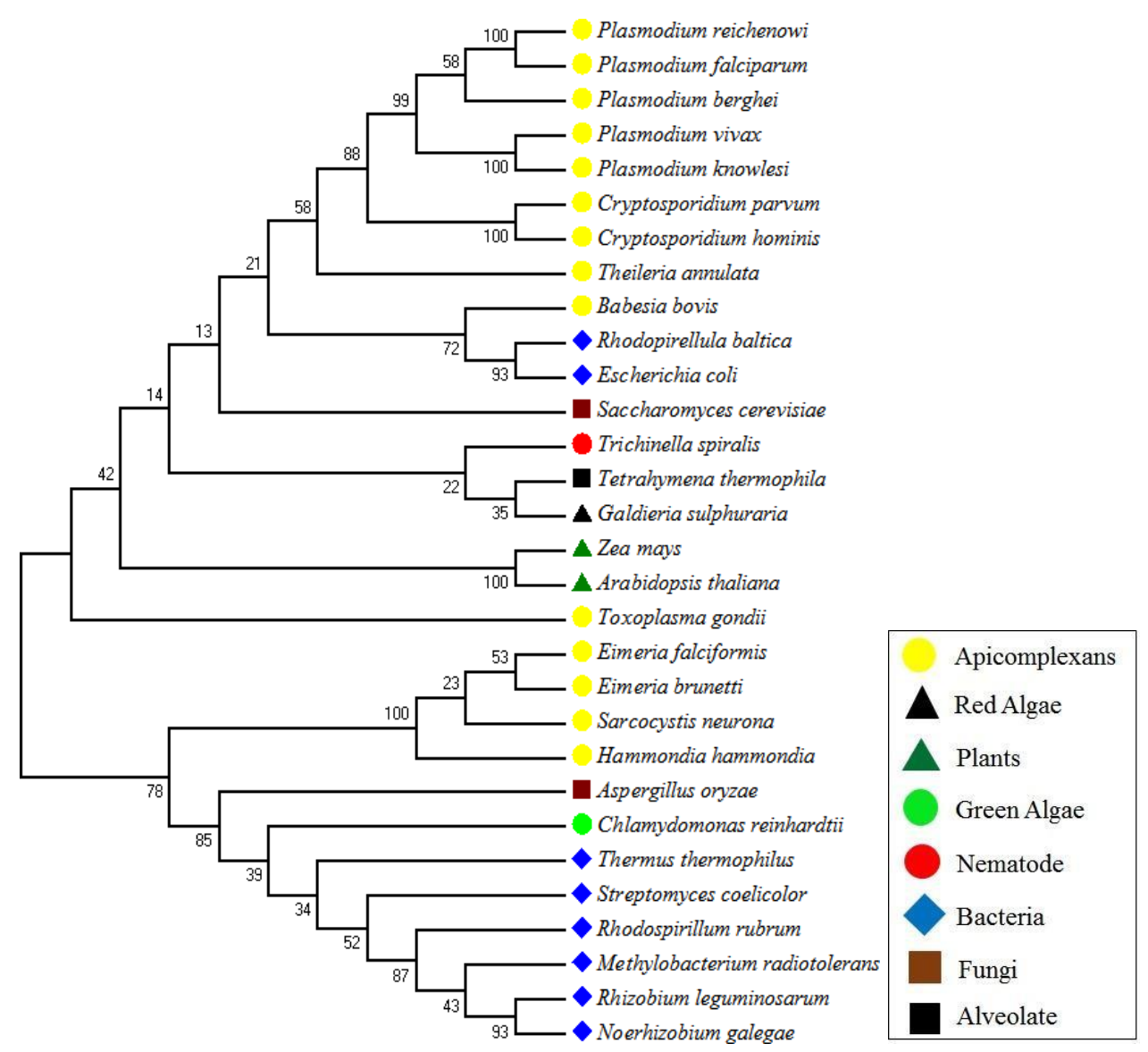

Figure 7. Phylogenetic relationship of PSY/OPPS gene in Plasmodium falciparum and other species.

Research has recently focused on the apicoplast, partially because this organelle is unique to the parasite and because it provides a fresh window into the parasite's metabolism (Goodman and McFadden 2013; Ralph et al. 2001). The carotenoid biosynthesis route is a fascinating metabolic route that occurs in the apicoplast and has gotten a lot of attention from scientists. The parasite has this route, but this route is absent in human (Tonhosolo et al. 2009). Fluridone, which inhibits phytoene desaturase in plants, is a well-known inhibitor of this process (McCowen et al. 1979). Other inhibitors of carotenoid biosynthesis, such as norflurazon and fosmidomycin, have been studied in the past and proven to be efficient at suppressing parasite growth in a dose-dependent manner in Plasmodium and Toxoplasma (Tonhosolo et al. 2009; Umeda et al. 2011; Yeh and DeRisi 2011). Fluridone's effect on Toxoplasma has also been examined, with results indicating that suppression caused a delay in egress and stimulated growth of the parasite's slow-growing, dormant cyst stage (Nagamune et al. 2008). However, the effect of fluridone on Plasmodium has not been explored. The goal of this research was to see how fluridone affected the carotenoid production pathway in $P$. falciparum.

Artemisinin was utilized as a control medicine in this research because its inhibitory capability against $P$. falciparum is known (Klonis et al. 2011). Fluridone was utilized as a comparison drug since its inhibitory capability against $P$. falciparum is unknown. The results of the inhibition assays (Figures 4.1 to 4.3 ) against the asexual stages revealed that fluridone was less effective than artemisinin at reducing parasite population, when comparing the concentrations at which each compound achieved its half-maximum inhibitory concentration against each stage. This could be owing to the incapability of fluridone to successfully traverse the membrane barriers surrounding the intraerythrocytic parasite, such as the RBC and parasite's lipid bilayers, as well as the parasitophorous vacuole encircling the parasite, preventing the molecule from reaching its intended target. The ring stage of the parasite life cycle was the most responsive to fluridone treatment of the three asexual stages because it had the lowest IC50 value $(17.00 \mathrm{mM})$ of the three asexual stages (Figure 1).

For an inhibitor to reach its target, it must be able to cross any barrier that stands between it and the target, such as enzymes and ligands, in sufficient numbers to disrupt or inhibit the target's activity (Clay and Sharom 2013). The plasma membrane is selectively permeable, allowing only certain chemicals to flow through while preventing others from doing so (Vaara, 1992). The inhibitor's physical and chemical features can help or hinder its passage across the phospholipid bilayer. Simple diffusion or osmosis allows small, non-polar (hydrophobic) substances like water, oxygen, and carbon dioxide to pass through the gaps in the 
membrane (Haines, 1994). Larger, charged molecules like glucose and amino acids, on the other hand, are transported across the membrane through active or passive diffusion through channels or pores (Stillwell 2013). Fluridone (Figure 2.4) is a fluorine-containing heterocyclic molecule that belongs to the nitrogen-containing heterocyclic class. Because this is a big molecule, it may require active or passive transport across the membrane via pores or channels. This could explain the high IC50 values obtained for fluridone treatment of the three intraerythrocytic stages of $P$. falciparum due to the restriction of fluridone mobility across membranes (parasitophorous vacuole and RBC and parasite membranes). The difference in the targets or modes of action of artemisinin and fluridone could also account for the high IC50 values seen for fluridone therapies. Artemisinin has been shown to inhibit hemoglobin uptake in $P$. falciparum intraerythrocytic stages (Klonis et al. 2011), whereas fluridone inhibits the activity of phytoene desaturase, the enzyme that catalyzes the conversion of phytoene to lycopene in the carotenoid biosynthesis pathway (Klonis et al. 2011 \& Tonhosolo et al. 2009). When phytoene desaturase is inhibited by fluridone, parasites may be able to salvage lycopene existing in the host blood circulation (which comes from ones diet), but proteins derived from hemoglobin digestion may not be salvaged.

\section{Carotenoid profiling and HPLC analyses}

Since the discovery of the apicoplast in 1996 (Mcfadden et al. 1996) and the association of certain pathways in apicomplexans, including the isoprenoid biosynthesis pathway (Tonkin et al. 2008), scientists have been interested in determining whether this organelle serves a function in this parasite group and could be targeted for chemotherapeutic management. In apicomplexans, the carotenoid biosynthesis pathway has been connected to the manufacture of isoprenoid precursors (Eisenreich et al. 2004). The condensation of two molecules of GGPP to generate phytoene, the first committed step of the carotenoid biosynthesis pathway, is catalyzed by phytoene synthase). Nagamune et al. (2008) were the first to show that carotenoids are synthesized and that inhibiting this pathway leads to development and growth inhibitions in apicomplexans (Toxoplasma). Tonhosolo and colleagues have also shown carotenoid production in $P$. falciparum intraerythrocytic stages (2009). However, only a few carotenoids have been found to be synthesized by $P$. falciparum, including $\beta$-carotene, phytoene, and lutein, leaving an incomplete picture of the carotenoid synthesis landscape in P. falciparum.

The types and quantities of carotenoids generated in three asexual stages of $P$. falciparum were studied in this study. The carotenoids were extracted using a ternary solvent solution that contained methanol, hexane, and water from synchronized $P$. falciparum cells at the ring, trophozoite, and schizont phases. Because carotenoids are soluble in organic solvents, they partition into the organic phase of the extraction solvent (methanol and hexane), which was then employed for reverse-phase HPLC analysis. The non-linear gradient was utilized to progressively decrease the polarity of the mobile phase by progressively increasing the volume of acetone to water in the mobile phase. This was done to allow non-polar chemicals (carotenoids, for example, and any other compounds removed) to elute later in the run. Because carotenoids have similar structures and could all elute together, the C-30 column is a favored column for successful separation. The C-30 column provides a greater number of interaction sites for the carotenoids to engage with the column than a C-18 column, ensuring complete partitioning of positional isomers, as occurs in carotenoids. The C-30 column increases the contact period of the eluents with the packing elements of the column, extending the retention period and considerably improving the selectivity of the carotenoid isomers (Breitenbach et al. 2001; Emenhiser et al. 1995).

Only one identifiable peak with retention time corresponding to lutein, and with one of the standards employed, was visible on the chromatogram for the ring stage extract (Figure 4). This could be because the carotenoids generated at this stage are so low that they are below detection limits. This shows that carotenoids' functions in the early stages of the parasite life cycle may be noncompulsory. Carotenoids protect organisms from oxidative damage by quenching photosensitizers, interacting with singlet oxygen molecules, and scavenging peroxy radicals. This then reduces the accumulation of hazardous oxygen species created during active metabolism, which is one of the major roles played by carotenoids in the organisms (Shimidzu et al. 1996; Stahl and Sies 2003). There are probably not enough free radicals present currently in the parasite's life cycle to cause activation and robust synthesis of these carotenoids in significant quantities, resulting in low levels of carotenoids at the ring stage.

Three peaks were found in the chromatogram for the trophozoite stage extract (Figure 5), compared to a single peak for the ring stage extract (Figure 4). Two of the peaks exhibited retention periods that matched the standards that were employed. $\alpha$-carotene (19.573 minutes) and $\beta$ carotene (30.303 minutes) were found as the peaks. The retention time of the third peak, which was 17.763 minutes, is unknown because it did not relate to any of the standards utilized in this study. However, because its retention duration falls within the range for carotenoids in our study, it is suspected to be a carotenoid. In comparison to the ring (Figure 4) and trophozoite (Figure 5), the schizont stage (Figure 6) generated several additional carotenoids. When comparing the schizont, ring, and trophozoite stages, it was discovered that the schizont stage had the highest quantities of carotenoids. This supports the findings of Tonhosolo et al. (2009), who found that carotenoid synthesis begins in the ring stages and builds up in the schizont stage. In their study, Tonhosolo et al. (2009) found carotenoids such as lutein and -carotene, which were also found in this study. Other carotenoids such as -carotene, apo-carotenal, lycopene, and abscisic acid were also profiled in $P$. falciparum for the first time in this study.

The presence and quantity of abscisic acid (with a retention period of 24.114 minutes) generated in the 
schizont stage was also discovered to be quite fascinating and interesting. Abscisic acid (ABA) is a phytohormone that controls a variety of critical plant processes, including stress responses, embryo development, and seed dormancy (Nakashima and Yamaguchi-Shinozaki 2013; Nambara et al. 2010). A surge in abscisic acid (ABA) levels signaled a calcium-dependent egress of the parasites from the host cells in T. gondii (Nagamune et al. 2008), a similar apicomplexan. But the inhibiting abscisic acid synthesis resulted in the formation of cysts, a dormant form of the parasite (Nagamune et al. 2008). Exogenous abscisic acid was shown to induce the formation of cyclic adenosine diphosphate ribose (cADPR, a second-messenger) in Toxoplasma gondii, stimulate calcium-dependent protein secretion, and induce parasite egress from the infected host cell in a density-dependent manner. This confirms the role of ABA in Toxoplasma. In addition, specific disruption of ABA production by the inhibitor fluridone delayed egress and induced formation of the Toxoplasma parasite's slowgrowing, latent cyst stage. In Toxoplasma, a closely related apicomplexan to Plasmodium, ABA-mediated calcium signaling governed the decision between lytic and chronic stage growth, a developmental switch that is critical in pathogenesis and transmission (Nagamune et al. 2008).

The absence of ABA in the ring and trophozoite stages was most likely due to the lack of or extremely low quantities of this phytohormone produced at these stages, which were below detection limits. However, there was an unexpected surge in ABA levels that was only found in the schizont stage. This could be the result of synthesis from the many individual merozoites still imprisoned in the parasitophorous vacuole. The discovery of an increase in abscisic acid levels in only the schizont stage is fascinating because it is the first time the phytohormone has been linked to $P$. falciparum. This means it could be serving the same crucial role in mediating parasite egress in this parasite as it is in $T$. gondii. To date, there has been no evidence of a signal for parasite egress from infected RBCs during the schizont stage, when merozoites are discharged to infect fresh RBCs. Whether or not ABA regulates or relates to merozoite egress in $P$. falciparum is unknown. Given that P. falciparum and $T$. gondii belong to the same Phylum Apicomplexa and share similar features such as the presence of the parasitophorous vacuole and the use of similar machinery for invading, it is possible to hypothesize that ABA may play a similar role in $P$. falciparum as it does in $T$. gondii, where it mediates parasite egress from its host cell (Kemp et al. 2013).

\section{Bioinformatics and phylogenetic analysis of the phytoene synthase gene}

Plasmodium falciparum, the most common Plasmodium species that infects people; it produces the most morbidity and mortality, with millions of clinical cases and over one million deaths reported each year (Greenwood et al. 2005; Hay et al. 2004). Although significant progress has been made in the management and control of $P$. falciparum (Kappe et al. 2010), the evolutionary origins and relatedness of this parasite and other Plasmodium diseases remain a source of debate and interest in recent years. Malaria phylogenetic research can aid in the development of new treatments and vaccines, as well as the understanding of host-pathogen interactions and the evolution of treatment resistance in the pathogen (Datta and Chauhan 2010). The chimpanzee parasite, $P$. reichenowi, has recently been shown to be the closest known relative of $P$. falciparum. This was thought to have diverged from its human counterpart, around the same time as the progenitors of chimps and humans, more than 5 million years ago (Escalante and Ayala, 1994; Jeffares et al. 2007; Rich et al. 1998). Different studies have found other Plasmodium strains in chimps, western gorillas, and bonobos in recent years. This suggests the probability that $P$. falciparum in humans emerged because of cross-species transmission from one or more of these apes (Rich et al. 2009; Krief et al. 2010; Prugnolle et al. 2010). Plasmodium species and other apicomplexan parasites have plastids, which suggests their fondness for cyanobacteria and green algae. Plasmodium species are assumed to be of prokaryotic origin since the apicoplast is produced through secondary endosymbiosis of an algal progenitor (McFadden 2011). Because the apicoplast lost its photosynthetic function in its new host, it kept certain prokaryotic remnants, the apicomplexan group of organisms is assumed to be connected to algae and plants (Foth and McFadden 2003; Arisue and Hashimoto 2014).

Plants, algae, and their endosymbiont apicomplexans have all been studied for isoprenoid and carotenoid production (Botella-Pava et al. 2004; Cunningham et al. 2007; Janoukovec et al. 2015). Isoprenoids and carotenoids are synthesized in apicomplexans via a separate mechanism (Goodman and McFadden 2013). The enzyme phytoene synthase (PSY), which catalyzes the first committed step of the isoprenoid to carotenoid biosynthesis pathway, is also found in apicomplexans parasites such as $P$. falciparum (Tonhosolo et al. 2009). Except for the PSY gene coding for the PSY enzyme, which has been identified through functional tests (Rodrguez-Villalón et al. 2009), no genetic evidence has been obtained for the enzymes assumed to be involved in the carotenoid biosynthesis pathway. Studies have looked at the structure and evolutionary relationship of the PSY gene in plants and other creatures (Giorio et al. 2008; Li et al. 2009; López-Emparán et al. 2014). This has recently been investigated in $P$. falciparum (Agarwal et al. 2015). The evolutionary relationship of the PSY gene in apicomplexans and other creatures is, however, poorly understood.

This study examined the evolutionary history and relationships of the phytoene synthase $(P S Y)$ gene in $P$. falciparum and other organisms. PSY orthologues were found in all Plasmodium species whose genome sequences were available, according to a BLAST search. Toxoplasma, Eimeria, Cryptosporidium, Babesia, and other apicomplexans employed in this phylogenetic analysis all had orthologues of this gene. However, because the apicomplexan species (shown by the yellow circles) bifurcate into two separate clades on the tree, all these orthologues in the apicomplexans were not closely related (Figure 7). Plasmodium, Cryptosporidium, Theileria, Toxoplasma, and Bebasia species formed a big 
monophyletic group with other species such as plants (green triangle) and algae (black triangle), and the other apicomplexans (Sarcocystis, Eimeria, and Hammondia species) formed another big clade. This shows a huge and substantial evolutionary event that generated a divergence in this gene; but most significantly, the $P S Y$ was still retained by the apicomplexans, implying that the $P S Y$ and carotenoid synthesis are crucial to the apicomplexan parasites.

Figure 10 shows that apicomplexan species (yellow circle) are members of a vast monophyletic group that also includes plants (green triangle) and algae (black triangle), supporting the theory that the apicoplast evolved from photosynthetic ancestry (McFadden 2011). All the Plasmodium species included in this study formed a subclade on a branch of the tree with a bootstrap value of 99, showing a high level of relatedness in the PSY sequences in this species. When taxa or species constitute a clade, it means they have a high level of relatedness when compared to members of other clades. Species that share a clade on a branch of the tree with a bootstrap value of 70 or higher indicate that there is a higher level of support for the existence or formation of that node, and the organisms that make up the node are likely to have shared a common ancestor at some point in their evolution (Soltis and Soltis 2003). With a bootstrap value of $100, P$. falciparum and $P$. reichenowi (the chimpanzee strain of Plasmodium) occupied the same clade as the other human strains employed in this investigation. This evidence backs with the theory that $P$. reichenowi is closest known relative of $P$. falciparum (Escalante and Ayala 1994; Jeffares et al. 2007).

In conclusion, the inhibition assays demonstrated that fluridone inhibited all three asexual stages of $P$. falciparum in a dose-dependent manner, with the ring stages being the most responsive to fluridone treatment. When compared to artemisinin, fluridone, a known plant carotenoid biosynthesis inhibitor, was not as effective at reducing parasite growth. This research also discovered that carotenoid production in the asexual stages is cumulative, beginning with the ring stage and ending with the schizont stage, with large quantities of abscisic acid created at this time. PSY orthologues were found in all the apicomplexan species analyzed using bioinformatics and phylogenetic analysis. It also revealed a significant amount of relatedness between the PSY gene in all the Plasmodium species examined. The $P S Y$ of $P$. falciparum is most like that of $P$. reichenowi, the chimpanzee malaria parasite, confirming the theory that $P$. falciparum is chimpanzeederived. Because the apicomplexans formed a monophyletic group with the plants and algae included in this study, the tree also supports the notion that the apicoplast has photosynthetic ancestry. The abovepresented data is significant because it sheds more light on the carotenoid biosynthesis pathway in P. falciparum and apicomplexans. This included the types and quantities of carotenoids synthesized and the compounds that affected the pathway. This study also strongly supports the notion that the pathway can be targeted for the control and possibly eradication of diseases caused by the parasite.

\section{REFERENCES}

Agarwal S, Sharma V, Phulera S, Abdin MZ, Ayana R, Singh S. 2015. Structural insights into a key carotenogenesis related enzyme phytoene synthase of $P$. falciparum: A novel drug target for malaria. Syst Synth Biol 9 (1): 27-37. DOI: 10.1007/s11693-015-9168-8.

Arisue N, Hashimoto T. 2014. Phylogeny and evolution of apicoplasts and apicomplexan parasites. Parasitol Int 64 (3): 254-259. DOI: 10.1016/j.parint.2014.10.005.

Bousema T, Drakeley C. 2011. Epidemiology and infectivity of Plasmodium falciparum and Plasmodium vivax gametocytes in relation to malaria control and elimination. Clin Microbiol Rev 24: 377-410. DOI: 10.1128/CMR.00051-10.

Breitenbach J, Braun G, Steiger S, Sandmann G. 2001. Chromatographic performance on a C30-bonded stationary phase of monohydroxycarotenoids with variable chain length or degree of desaturation and of lycopene isomers synthesized by various carotene desaturases. J Chromatograph A 936 (1-2): 59-69. DOI: 10.1016/S0021-9673(01)00945-1.

Casares S, Brumeanu TD, Richie TL. 2010. The RTS,S malaria vaccine. Vaccine 28 (31): 4880-4894. DOI: 10.1016/j.vaccine.2010.05.033.

Choi S-R, Mukherjee P, Avery MA. 2008. The fight against drug-resistant malaria: Novel plasmodial targets and antimalarial drugs. Curr Med Chem 15 (2): 161-171. DOI: 10.2174/092986708783330575.

Clay AT, Sharom FJ. 2013. Lipid bilayer properties control membrane partitioning, binding, and transport of P-glycoprotein substrates. Biochem 52 (2): 343-354. DOI: 10.1021/bi301532c.

Crompton PD, Pierce SK, Miller LH. 2010. Advances and challenges in malaria vaccine development. J Clin Investig 120 (12): 4168-4178. DOI: $10.1172 / J C I 44423$.

Crowther GJ, Napuli AJ, Gilligan JH, Gagaring K, Borboa R, Francek C, Chen Z, Dagostino EF, et al. 2011. Identification of inhibitors for putative malaria drug targets among novel antimalarial compounds. Mol Biochem Parasitol 175 (1): 21-29. DOI: 10.1016/j.molbiopara.2010.08.005.

Cunningham FX, Lee H, Gantt E. 2007. Carotenoid biosynthesis in the primitive red alga Cyanidioschyzon merolae. Eukaryotic Cell 6 (3): 533-545. DOI: 10.1128/EC.00265-06.

Datta N, Chauhan VS. 2010. Origin and evolution of human malaria parasite, P. falciparum and P. vivax. In: Sharma VP. (Editor). Nature at Work: The Ongoing Saga of Evolution. Springer, India.

Djimdé A, Doumba OK, Cortese JF, Kayentao K, Doumbo S, Diourte Y, Dicko A, Xin-Zhuan S, Takashi N, Fidock DA, Wellems TE, Plowe CV. 2001. A molecular marker for chloroquine-resistant falciparum malaria. New England J Med 344 (4): 257-263. DOI: 10.1056/NEJM200101253440403.

Dondorp AM, Yeung S, White L, Nguon C, Day NPJ, Socheat D, von Seidlein L. 2010. Artemisinin resistance: Current status and scenarios for containment. Nat Rev Microbiol 8 (4): 272-280. DOI: 10.1038/nrmicro2331.

Emenhiser C, Sander LC, Schwartz SJ. 1995. Capability of a polymeric C30 stationary phase to resolve cis-trans carotenoid isomers in reversed-phase liquid chromatography. J Chromatograph A 707 (2): 205-216. DOI: 10.1016/0021-9673(95)00336-L.

Escalante AA, Ayala FJ. 1994. Phylogeny of the malarial genus Plasmodium, derived from rRNA gene sequences. Proc National Acad Sci USA 91 (24): 11373-11377. DOI: 10.1073/pnas.91.24.11373.

Foth BJ, McFadden GI. 2003. The apicoplast: A plastid in Plasmodium falciparum and other apicomplexan parasites. Int Rev Cytol 224: 57110. DOI: 10.1016/S0074-7696(05)24003-2.

Giorio G, Stigliani AL, D`Ambrosio C. 2008. Phytoene synthase genes in tomato (Solanum lycopersicum L.) - new data on the structures, the deduced amino acid sequences, and the expression patterns. FEBS J 275 (3): 527-35. DOI: 10.1111/j.1742-4658.2007.06219.x.

Goodman CD, McFadden GI. 2013. Targeting apicoplasts in malaria parasites. Expert Opinion on Therapeutic Targets 17 (2): 167-77. DOI: $10.1517 / 14728222.2013 .739158$.

Gornicki P. 2003. Apicoplast fatty acid biosynthesis as a target for medical intervention in apicomplexan parasites. Int J Parasitol 33 (9): 885-896.DOI: 10.1016/s0020-7519(03)00133-4.

Greenwood BM, Bojang K, Whitty CJ, Targett GA. 2005. Malaria. Lancet 365 (9469): 1487-1498. DOI: 10.1016/S0140-6736(05)664203. 
Haines TH. 1994. Water transport across biological membranes. FEBS Lett 346 (1): 115-122. DOI: 10.1016/0014-5793(94)00470-6.

Hay SI, Guerra CA, Gething PW, Patil AP, Tatem AJ, Noor AM, Kabaria CW, Manh BH, Elyazar IRF, Brooker S, Smith DL, Moyeed RA, Snow RW. 2009. A world malaria map: Plasmodium falciparum endemicity in 2007. PLoS Med 6 (3): e1000048. DOI: 10.1371/journal.pmed.1000048.

Hay SI, Guerra CA, Tatem AJ, Noor AM, Snow RW. 2004. The global distribution and population at risk of malaria: Past, present, and future. Lancet Infect Dis 4 (6): 327-336. DOI: 10.1016/S14733099(04)01043-6.

Jeffares DC, Pain A, Berry A, Cox AV, Stalker J, Ingle CE, Thomas A, Quail MA, Siebenthal K, Uhlemann AC, Kyes S, Krishna S, Newbold C, Dermitzakis ET, Berriman M. 2007. Genome variation and evolution of the malaria parasite Plasmodium falciparum. Nat Genet 39 (1): 120-125. DOI: 10.1038/ng1931.

Jiang N, Chang Q, Sun X, Lu H, Yin J, Zhang Z, Wahlgren M, Chen Q. 2010. Co-infections with Plasmodium knowlesi and other malaria parasites, Myanmar. Emerg Infect Dis 16: 1476-1478. DOI: 10.3201/eid1609.100339.

Jordão FM, Kimura EA, Katzin AM. 2011. Isoprenoid biosynthesis in the erythrocytic stages of Plasmodium falciparum. Memórias Do Instituto Oswaldo Cruz 106 (1): 134-141. DOI: 10.1590/S007402762011000900018.

Kalanon M, McFadden GI. 2010. Malaria, Plasmodium falciparum and its apicoplast. Biochem Soc Transactions 38 (3): 775-782. DOI: 10.1042/BST0380775

Kappe SHI, Vaughan AM, Boddey JA, Cowman AF. 2010. That was then but this is now: malaria research in the time of an eradication agenda. Science 328 (5980): 862-866. DOI: 10.1126/science.1184785.

Kemp LE, Yamamoto M, Soldati-Favre D. 2013. Subversion of hos cellular functions by the apicomplexan parasites. FEMS Microbiol Rev 37 (4): 607-631. DOI: 10.1111/1574-6976.12013.

Klonis N, Crespo-Ortiz MP, Bottova I, Abu-Bakar N, Kenny S, Rosenthal PJ, Tilley L. 2011. Artemisinin activity against Plasmodium falciparum requires hemoglobin uptake and digestion. Proc National Acad Sci USA 108 (28): 11405-11410. 10.1073/pnas.1104063108

Krief S, Escalante AA, Pacheco MA, Mugisha L, André C, Halbwax M, Fischer A, Krief JM, Kasenene JM, Cranfield M, Cornejo OE, Chavatte JM, Lin C, Letourneur F, Grüner AC, McCutchan TF, Rénia L, Snounou G. 2010. On the diversity of malaria parasites in African apes and the origin of Plasmodium falciparum from Bonobos. PLoS Pathogens 6 (2): e1000765. DOI: 10.1371/journal.ppat.1000765.

Lambros C, Vanderberg JP. 1979. Synchronization of Plasmodium falciparum erythrocytic stages in culture. J Parasitol 65 (3): 418-420. DOI: $10.2307 / 3280287$.

Li F, Tsfadia O, Wurtzel ET. 2009. The phytoene synthase gene family in the Grasses. Plant Signal Behav 4 (3): 208-211. DOI: 10.4161/psb.4.3.7798.

López-Emparán A, Quezada-Martinez D, Zúñiga-Bustos M, Cifuentes V, Iñiguez-Luy F, Federico ML. 2014. Functional Analysis of the Brassica napus L. Phytoene Synthase (PSY) Gene Family. PloS One 9 (12): e114878. DOI: 10.1371/journal.pone.0114878.

Maier AG, Rug M. 2013. In vitro culturing Plasmodium falciparum erythrocytic stages. Method Mol Biol 923: 3-15. DOI: 10.1007/978-162703-026-7 1.

malERA Consultative Group on Vaccines. 2011. A research agenda for malaria eradication: Vaccines. PLoS Med 8 (1): e1000398. DOI: 10.1371/journal.pmed.1000398.

Malik S, Gupta M, Braich J. 2012. Current scenario of malaria vaccine. Int J Basic Clin Pharmacol 1 (2): 60-66. DOI: 10.5455/2319 2003.ijbcp002112.

Maréchal E, Cesbron-Delauw MF. 2001. The apicoplast: A new member of the plastid family. Trends Plant Sci 6 (5): 200-205. DOI: 10.1016/S1360-1385(01)01921-5.

McCowen MC, Young CL, West SD, Parka SJ, Arnold WR. 1979. Fluridone, a new herbicide for aquatic plant management. J Aquat Plant Manage 17: 27-30.

McFadden GI. 2011. The apicoplast. Protoplasma 248 (4): 641-650. DOI 10.1007/s00709-010-0250-5.

Nagamune K, Hicks LM, Fux B, Brossier F, Chini EN, Sibley LD. 2008 Abscisic acid controls calcium-dependent egress and development in Toxoplasma gondii. Nature 451: 207-210. DOI: 10.1038/nature06478.

Nakashima K, Yamaguchi-Shinozaki K. 2013. ABA signaling in stressresponse and seed development. Plant Cell Rep 32 (7): 959-970. DOI: 10.1007/s00299-013-1418-1.
Nambara E, Okamoto M, Tatematsu K, Yano R, Seo M, Kamiya Y. 2010. Abscisic acid and the control of seed dormancy and germination. Seed Sci Res 20 (2): 55-67. DOI: 10.1017/S0960258510000012.

Paniagua-Michel J, Olmos-Soto J, Ruiz MA. 2012. Pathways of carotenoid biosynthesis in bacteria and microalgae. Methods Mol Biol 892: 1-12. DOI: 10.1007/978-1-61779-879-5 1.

Perlmann P, Troye-Blomberg M. 2000. Malaria blood-stage infection and its control by the immune system. Folia Biologica 46: 210-218.

Prugnolle F, Durand P, Neel C, Ollomo B, Ayala FJ, Arnathau C, Etienne L, Mpoudi-Ngole E, Nkoghe D, Leroy E, Delaporte E, Peeters M, Renaud F. 2010. African great apes are natural hosts of multiple related malaria species, including Plasmodium falciparum. Proc National Acad Sci USA 107 (4): 1458-1463. DOI: 10.1073/pnas.0914440107.

Qidwai T, Khan F. 2012. Antimalarial drugs and drug targets specific to fatty acid metabolic pathway of Plasmodium falciparum. Chemical Biol Drug Design 80 (2): 155-172. DOI: 10.1111/j.17470285.2012.01389.x

Raghavendra K, Barik TK, Reddy BPN, Sharma P, Dash AP. 2011. Malaria vector control: from past to future. Parasitol Res 108 (4): 757-779. DOI: 10.1007/s00436-010-2232-0.

Ralph SA, D`Ombrain MC, McFadden GI. 2001. The apicoplast as an antimalarial drug target. Drug Resist Updat 4 (3): 145-511. DOI: 10.1054/drup.2001.0205.

Rich SM, Leendertz FH, Xu G, LeBreton M, Djoko CF, Aminake MN, Takang EE, Diffo JLD, Pike BL, Rosenthal BM, Formenty P, Boesch C, Ayala FJ, Wolfe ND. 2009. The origin of malignant malaria. Proc National Acad Sci USA 106 (35): 14902-14907. DOI: 10.1073/pnas.0907740106.

Rich SM, Licht MC, Hudson RR, Ayala FJ. 1998. Malaria‘s Eve: Evidence of a recent population bottleneck throughout the world populations of Plasmodium falciparum. Proc National Acad Sci USA 95 (8): 4425-4430. DOI: 10.1073/pnas.95.8.4425.

Rieckmann KH. 2006. The chequered history of malaria control: are new and better tools the ultimate answer? Annals Trop Med Parasitol 100 (8): 647-662. 10.1179/136485906X112185.

Rodríguez-Concepción M, Boronat A. 2013. Isoprenoid Synthesis in Plants and Microorganisms. In: Bach TJ, Rohmer M. (Editors), Isoprenoid Synthesis in Plants and Microorganisms: New Concepts and Experimental Approaches. Springer, New York

Sato S. 2011. The apicomplexan plastid and its evolution. Cell Mol Life Sci 68 (8): 1285-1296. DOI: 10.1007/s00018-011-0646-1.

Schellenberg D, Abdulla S, Roper C. 2006. Current issues for antimalarial drugs to control $P$. falciparum malaria. Curr Mol Med 6 (2): 253- 260. DOI: 10.2174/156652406776055168.

Schwartz L, Brown GV, Genton B, Moorthy VS. 2012. A review of malaria vaccine clinical projects based on the WHO rainbow table. Malar J 11: 11. DOI: 10.1186/1475-2875-11-11.

Seeber F. 2003. Biosynthetic pathways of plastid-derived organelles as potential drug targets against parasitic apicomplexa. Curr Drug Targets Immune Endocr Metabol Disord 3 (2): 99-109. DOI: $10.2174 / 1568008033340261$.

Seeber F, Soldati-Favre D. 2010. Metabolic pathways in the apicoplast of apicomplexa. Int Rev Cell Mol Biol 281: 161-228. DOI: 10.1016/S1937-6448(10)81005-6.

Sermwittayawong N, Singh B, Nishibuchi M, Sawangjaroen N, Vuddhakul V. 2012. Human Plasmodium knowlesi infection in Ranong province, southwestern border of Thailand. Malaria J 11: 36. DOI: $10.1186 / 1475-2875-11-36$.

Sharma R, Dutta AK. 2011. Malaria and national vector-borne disease control programme. Indian J Pediatrics 78: 1527-1535. DOI: 10.1007/s12098-011-0554-2.

Shiff C. 2002. Integrated Approach to Malaria Control. Clin Microbiol Rev 15: 278-293. DOI: 10.1128/CMR.15.2.278-293.2002.

Shimidzu N, Goto M, Miki W. 1996. Carotenoids as singlet oxygen quenchers in marine organisms. Fisheries Sci 62 (1): 134-137. DOI: 10.2331/fishsci.62.134

Soltis DE, Soltis PS. 2003. Applying the bootstrap in phylogeny reconstruction. Statistical Sci 18 (2): 256-267. DOI: $10.1214 / \mathrm{ss} / 1063994980$.

Stahl W, Sies H. 2003. Antioxidant activity of carotenoids. Mol Aspects Med 24 (6): 345-351. DOI: 10.1016/S0098-2997(03)00030-X

Stillwell W. 2013. Membrane Transport. An Introduction to Biological Membranes. Elsevier B. V., Amsterdam.

Toledo-Ortiz G, Huq E, Rodríguez-Concepción M. 2010. Direct regulation of phytoene synthase gene expression and carotenoid 
biosynthesis by phytochrome- interacting factors. Proc National Acad Sci USA 107 (25): 11626-11631. DOI: 10.1073/pnas.0914428107.

Tonhosolo R, D‘Alexandri FL, de Rosso VV, Gazarin ML, Matsumura MY, Peres VJ, Merino EF, Carlton JM, Wunderlich G, Mercadante AZ, Kimura EA, Katzin AM. 2009. Carotenoid biosynthesis in intraerythrocytic stages of Plasmodium falciparum. J Biol Chem 284 (15): 9974-9985. DOI: 10.1074/jbc.M807464200.

Tonhosolo R, D‘Alexandri FL, Genta FA, Wunderlich G, Gozzo FC, Eberlin MN, Peres VJ, Kimura EA, Katzin AM. 2005. Identification, molecular cloning, and functional characterization of an octaprenyl pyrophosphate synthase in intra-erythrocytic stages of Plasmodium falciparum. Biochem J 392 (1): 117-126. DOI: 10.1042/BJ20050441.

Tonkin CJ, Kalanon M, McFadden GI. 2008. Protein targeting to the malaria parasite plastid. Traffic 9: 166-175.

Trager W, Jensen JB. 1976. Human malaria parasites in continuous culture. Science 193 (4254): 673-675. DOI: 10.1126/science.781840

Umeda T, Tanaka N, Kusakabe Y, Nakanishi M, Kitade Y, Nakamura KT 2011. Molecular basis of fosmidomycin's action on the human malaria parasite Plasmodium falciparum. Sci Rep 1: 9. DOI: 10.1038/srep00009.
Vaara M. 1992. Agents that increase the permeability of the outer membrane. Microbiol Rev 56 (3): 395-411. DOI: 10.1128/MMBR.56.3.395-411.1992.

van der Meer JY, Hirsch AKH. 2012. The isoprenoid-precursor dependence of Plasmodium spp. Nat Prod Rep 29: 721. DOI: 10.1039/c2np20013a.

Welsch R, Beyer P, Hugueney P, Kleinig H, von Lintig J. 2000. Regulation and activation of phytoene synthase, a key enzyme in carotenoid biosynthesis, during photomorphogenesis. Planta 211 (6): 846-854. 10.1007/s004250000352.

Wiesner J, Borrmann S, Jomaa H. 2003. Fosmidomycin for the treatment of malaria. Parasitol Res 90 (2): 71-76. DOI: 10.1007/s00436-0020770-9.

World Health Organisation. 2014. World Malaria Report 2014. World Health Organisation, Geneva.

Wright GJ, Rayner JC. 2014. Plasmodium falciparum Erythrocyte Invasion: Combining Function with Immune Evasion. PLoS Pathogens 10 (3): e1003943. DOI: 10.1371/journal.ppat.1003943.

Yeh E, DeRisi JL. 2011. Chemical rescue of malaria parasites lacking an apicoplast defines organelle function in blood-stage Plasmodium falciparum. PLoS Biol 9 (8): e1001138. DOI: 10.1371/journal.pbio.1001138. 
Table S1. Table showing the gene ID of PSY/OPPS in the various organisms used for the phylogenetic relationship study

\begin{tabular}{|c|c|c|}
\hline Organism & Gene ID & Database \\
\hline Plasmodium falciparum 3D7 & PF3D7_0202700, PFB0130w & PlasmoDB, NCBI \\
\hline Plasmodium vivax & PVX_003575 & PlasmoDB \\
\hline Plasmodium berghei ANKA & PBANKA_0300800 & PlasmoDB \\
\hline Plasmodium reichenowi & gi|832044551 & PlasmoDB \\
\hline Plasmodium knowlesi strain H & PKNH_0418400 & PlasmoDB \\
\hline Cryptosporidium hominis TU502 & cgd5_4532 & ToxoDB \\
\hline Cryptosporidium parvum Iowa II & cgd7_3730 & ToxoDB \\
\hline Eimeria brunette & EBH_0010260 & ToxoDB \\
\hline Eimeria falciformis & EfaB_MINUS_800.g99_1 & ToxoDB \\
\hline Hammondia strain H.H.34 & HHA_224490 & ToxoDB \\
\hline Theileria annulata & TA035 05 & ToxoDB \\
\hline Toxoplasma gondii & TGME49_269430 & ToxoDB \\
\hline Sarcocystis neurona & SN3_00400385 & ToxoDB \\
\hline Arabidopsis thaliana & gi|240256493 & NCBI \\
\hline Aspergillus oryzae & gi|169781061 & NCBI \\
\hline Babesia bovis T2Bo & gi| 156087461 & NCBI \\
\hline Chlamydomonas reinhardtii & gi|159486413 & NCBI \\
\hline Escherichia coli & gi|387615344 & NCBI \\
\hline Galdieria sulphuria & gi|545700663 & NCBI \\
\hline Methylobacterium radiotolerans & gi| 170746450 & NCBI \\
\hline Neorhizobium galegae & gi|752716557 & NCBI \\
\hline Rhizobium leguminosarum & gi|752843554 & NCBI \\
\hline Rhodopirellula baltica & gi|32470666 & NCBI \\
\hline Rhodospirillum rubrum & gi| 83591340 & NCBI \\
\hline Saccharomyces cerevisiae & gi| 330443520 & NCBI \\
\hline Streptomyces coelicolor & gi|32141095 & NCBI \\
\hline Tetrahymena thermophila & gi|229594551 & NCBI \\
\hline Thermus thermophilus & gi| 593268528 & NCBI \\
\hline Trichinella spiralis & gi|331705224 & NCBI \\
\hline
\end{tabular}

